\title{
Automatic evaluation and improvement of roof segments for modelling missing details using Lidar data
}

Fayez Tarsha Kurdi* and Mohammad Awrangjeb

Institute for Integrated and Intelligent Systems, Griffith University, Nathan QLD 4111, Australia

(f.tarshakurdi, m.awrangjeb)@griffith.edu.au 


\title{
Automatic evaluation and improvement of roof segments for modelling missing details using Lidar data
}

\begin{abstract}
Despite the large number of studies conducted during the last three decades concerning 3D building modelling starting from Lidar data, two persistent problems still exist. The first one is the absence of some roof details which will not only disappear in the building roof model due to their small areas regarding the point density, but are also considered as undesirable noise among the modelling procedures. The second problem consists in that the involved segmentation algorithms do not perform well in the presence of noise in the building point cloud data. These two problems generate undesirable deformation in the final 3D building model. This paper proposes a new automatic approach for detecting and modelling the missing roof details in addition to improving the building roof segments. In this context, the error map matrix, which presents the deviations of points to their fitting planes, is considered. Moreover, this matrix is analyzed in order to deduce the mask of missing roof details. At this stage, a new numeric factor is defined for estimating the roof segmentation accuracy in addition to the validity of the roof segmentation result. Then, the building point cloud is enhanced in order to decrease the negative noise influence and, consequently, to improve the building roof segments. Finally, the functionality and the accuracy of the proposed approach are tested and discussed.
\end{abstract}

Key words: Lidar, modelling, segmentation, missing details, point cloud

\section{Introduction}

The demand for digital 3D urban models continues to grow. This can be explained by the ease of use and the immediate understandability of such models compared to 2D models. In this context, Lidar technique automatically provides a Digital Surface Model (DSM) of the scanned area. Even if the volume of data is huge, this type of data has quickly been approved especially as a support for GIS databases, decision-making, urban and environmental planning. After the acquisition of 3D laser data in urban areas, a 3D model can be generated after the 3D point cloud data have been segmented into three main classes: terrain, vegetation and buildings. Once 
airborne laser scanning technology became popular, it was realized that the modelling of roof planes and their topological relationships are essential for producing 3D building models. Thereby, significant efforts have been made towards this goal.

In this context, building modelling using Lidar data means the calculation of a 3D building model composed of roof surfaces, edge-lines and vertices, starting from the point cloud covering spatially the building roof. Despite the fact that building roofs sometimes contain no planar surfaces such as domes, cones, cylinders, or mathematical curved surfaces, the basic hypothesis adopted in building modelling approaches is that the building roof is composed of a set of planes. These planes are connected in describing roof geometry. Starting from this hypothesis, building roof planes can be classified into three classes. The first class is the principal planes that cover most of the roof area. The second class is the vertical planes that present in step edge connections. The final class is the roof details such as chimneys and windows. The last class can also be subdivided into two subclasses having considerable altitudes but are different in the area value. The first subclass presents the detail planes, which have negligible areas regarding to the point density, and therefore, it is hard to detect them (missing details). The second subclass presents the detail planes, which have considerable areas regarding to the point density and, therefore, they are detected easily (detectable details).

At this stage, it is important to note that the missing details present various objects that can be located on a building roof such as chimneys, windows, antennas, tanks, decorative features, or obstacles that may be found there. Thus, they have irregular geometric forms. Moreover, the number of points which represent these details is negligible, which is why they cannot be detected during roof segmentation and, thereby, are considered as noise. Furthermore, other noisy points may occur in their neighbourhood, which can be explained by the diameter of the 
laser spot, which generates a planimetric error, in addition to the scanned points of vertical elements surrounding these details. Consequently, the geometric form of the 'missing details' is still vague in the point cloud. However, considering the basic hypothesis, which states that the building roof is composed of a set of planes, and regarding the goal of this study, which is the detection and the modelling of the missing details in $2 \mathrm{D}$, these details will be modelled by means of small square planes to complete the 2D model of the roof.

As it is well known in mathematics of geometry, a line presents the projection of the vertical plane onto a horizontal coordinate system. Moreover, the missing detail planes will not only disappear in the $2 \mathrm{D}$ roof model due to their small areas, but they will be considered as undesirable noise among modelling procedures. Thus, the 2D building roof model will only contain two types of planes: principal planes and detectable detail planes.

Regarding the missing planes, the contributions of this paper can be summarized as follows:

- Detection and modelling the missing detail planes that were considered in the past as undesirable noise because of their small areas in comparison to the point density.

- Definition of a new numeric factor called Segmentation Accuracy and Quality (SAQ) factor. This factor estimates the roof segmentation validity and quality and plays an essential role in acceptance or rejection of building roof segmentation outcomes.

- Suggestion of a new approach for automatic improvement of building roof segments.

The organigram presented in Figure 1 exposes the consecutive steps of the proposed approach. The three precedent operations allow control, enhancement and improvement of the 2D building model before starting the calculation of the 3D building model. 
Once the $2 \mathrm{D}$ building model is calculated accurately, the way towards constructing the $3 \mathrm{D}$ building model will be paved.

\section{Related Work}

The subject of this paper contributes to the building roof segmentation topic, which is covered widely in the literature. The suggested approaches can be grouped into the following six families:

(1) Approaches using the RANdom SAmpling Consensus paradigm (RANSAC): For instance, Brenner (2000), Salehi and Mohammadzadeh (2017), Demir (2018), and Wen et al. (2019) applied the RANSAC algorithm for detecting the roof planes. Forlani et al. (2006) applied the RANSAC paradigm for a dual purpose: to correct the roof segmentation result and to carry out the $2 \mathrm{D}$ segmentation of the building outline polygon. Furthermore, Tarsha Kurdi et al. (2008) extended its use for obtaining the mathematical model consistent with the geometry of a building roof. Bretar and Roux (2005) used the Normal Driven RANSAC (ND-RANSAC) for extracting 3D planar primitives. To achieve this, they calculated the normal vectors for each point before searching the roof planes randomly.

(2) Approaches using the Hough-Transform (HT): Vosselman and Dijkman (2001), Oda et al. (2004), Overby et al. (2004) and Tarsha Kurdi et al. (2007) used the 3D HT algorithm for detecting the roof planes. Moreover, Rabbani and Van den Heuvel (2005) used an improved HT algorithm for extraction of other 3D geometric forms like cylindrical objects. In the same context, Hofmann (2004) used the HT algorithm to analyze the tin-structure in the parameter space. 
Approaches using region growing algorithm: Alharthy and Bethel (2004), and Elaksher and Bethel (2002) developed an algorithm that gathers all pixels fitting a plane in raster data. Rottensteiner (2003) extracted roof planes by using seed regions and applied a region-growing algorithm in a regularized DSM. Then, the homogeneity relationships between the neighbour points are evaluated by calculating point normals. Awrangjeb et al. (2018) applied a new region-growing algorithm for detecting the roof planes. Furthermore, the missing planes were identified through an analysis using the 3D plane intersection lines between adjacent planes. Sohn et al. (2008) applied the region growing and TIN analysis for extracting roof planes. Then, the HT algorithm and neighbouring plane intersection lines were applied in order to extract plane boundaries.

(4) Approaches using model-driven tactic: Several authors Park et al. (2006) and Kadaa and McKinley (2009) suggested segmenting the complex building point cloud into primitives using the ground plans or similar type of meta data. Xiong et al. (2015) suggested a model-driven approach based on finding the roof topology graphs. These graphs have three basic elements: loose nodes, loose edges, and minimum cycles. A building is decomposed into a group of building parts predefined in a given library of building primitives. Then, determined constraints are applied in order to simultaneously adjust all roofs. Zhang et al. (2014) suggested representing building roofs by geometric primitives and constructing a cost function by using the constraints from both Lidar data and aerial imagery simultaneously. Zheng et al. (2017) proposed a hybrid approach of data- and model-driven approaches, in which Lidar data, 2D building footprint and a high-resolution orthophoto were used. The process started with the extraction of step edge lines on the building roof using Lidar and 2D building footprints by using the Canny 
filter. Then, three types of sub-footprints were created, for each one a roof model selection procedure was performed to match the primitives stored in the predefined library.

Approaches using aerial or satellite images in addition to Lidar data: Schenk and Csatho (2002) detected roof plane boundaries from panchromatic images based on the texture discontinuity and utilized them to refine the results from Lidar data. Sohn and Dowman (2007) used the multispectral images for roof plane boundary detection. Rottensteiner (2010) used multiple aerial images and a point cloud for detecting roof planes. It started with a building segmentation of each of the images, and thereafter the point cloud was used to determine which image segments correspond to planes.

(6) Other approaches: One approach suggested by Ghaffarian et al. (2016) utilized the Purposive Fast Independent Component Analysis algorithm (PFICA) for detecting roof plane boundaries, then Douglas-Peucker algorithm Douglas and Peucker (1973) was used after enhancing the obtained result by morphological filter. Sampath and Shan (2006) detected roof planes and plane boundaries simultaneously by using Lidar data exclusively. They applied the linear space theory to separate the plane boundaries from planar points. Xiao et al. (2015) started by extracting roof planes using a Gaussian mixture model in which buildings were presented by a mixture of Gaussians. Cao et al. (2017) applied a Density-Based Spatial Clustering of Applications with Noise (DBSCAN) in order to segment the building point sets into individual buildings with arbitrary shapes. Park et al. (2019) proposed a segmentation method based on a 3D shape descriptor to recognize objects. In this context, the same cube codes were gathered 
together as a segmented region. Then, the cube operator was used to segment building point cloud by classifying the coded surface slopes.

Before proceeding to the central idea of this paper, it is important to note that most of these previously cited approaches handle the missing roof details as undesirable noise. In the case of 3D point cloud obtained by a multi-view dense matching, the first envisaged operation is the building extraction from a city point cloud (Acar et al., 2019). Once the building point cloud is extracted, the next step is the 3D building modelling. In this context, Bulatov and Pohl (2013) developed an approach for detecting the small roof details in image sequences. Assumptions were applied in order to tighten the search range for chimneys and to reduce the number of false alarms. Finally, additional filtering was applied by means of colour or intensity images.

In the next section, the used datasets are presented.

\section{Data}

In order to test our approach on different point densities, three data sets are used in this paper (see Table 1).

\section{[Insert Table 1 here]}

The first data site 'Hermanni' is a residential area on the outskirts of Helsinki, where large and spaced storey houses are surrounded by vegetation. This point cloud belongs to the building extraction project of EuroSDR (www.eurosdr.org). The second point cloud is of the city of Strasbourg in France. Several zones with different typology natures are covered. However, point density and accuracy are lower in comparison to the Hermanni point cloud. The third dataset is captured over the city of Vaihingen in Germany (Cramer, 2010). This area is characterized by a few residential buildings, historic buildings and small-detached houses that are surrounded by 
trees.

\section{Building roof segmentation}

In order to extract the roof planes automatically from the Lidar data, several approaches are suggested in the literature. Most of these approaches allow calculating building label image, which presents the segmented building roof in $2 \mathrm{D}$ according to its composing planes. In this paper, the extended RANSAC algorithm suggested by Tarsha Kurdi et al. (2008) is employed. Because it allows coordination of the mathematical aspect of the RANSAC algorithm with the geometry of a roof. The input data of this algorithm is the 3D building point cloud, its output is the building label image (Figure $2 a$ ). Once the building point cloud is segmented successfully, the next step is the calculation of the $2 \mathrm{D}$ building model. For achieving this purpose, it is started by analyzing the adjacency relationship between roof planes. This step allows the determination of neighbouring planes. Then, the inner roof plane boundaries are detected and each one is given a specific number. Finally, the junction relationships between roof plane boundaries are analyzed in order to detect the roof vertices (Tarsha Kurdi et al., 2019). Figure $2 b$ shows the calculated 2D building model starting from building label image presented in Figure $2 a$.

\section{[Insert Figure 2 here]}

After constructing the 2D building model, it is necessary to estimate the segmentation accuracy; hence, the error map matrix is calculated (Tarsha Kurdi et al., 2019). This matrix facilitates 'pixel by pixel' analysis of deformations and the accuracy of the segmentation of building roof. Consequently, it permits the accuracy of constructed building model to be evaluated. For calculating this matrix, the distance (deviation) between each point and its mean plane is calculated and assigned to the corresponding pixel. Finally, regarding the small areas of detectable roof details and their complex geometric forms, the pixels of detectable detail planes 
will not be considered. That means, in the error map matrix, zero value will be assigned to pixels of detectable details without calculating the deviations. In order to visualize the error map matrix, we map the deviations greater than $+1 \mathrm{~m}$ to $+1 \mathrm{~m}$, and the deviations smaller than $-1 \mathrm{~m}$ to $-1 \mathrm{~m}$ (see Figure $3 a$ ). Therefore, all pixel values in this matrix are between +1 and -1 . Section 8 discusses on the choice of parameter values used in the proposed method.

\section{Analysis of error map matrix}

In the previous section, it is shown that in the error map matrix, the pixel values are between -1 and +1 . The calculation of the histogram of pixel values in the error map matrix (Figure $3 b$ ) shows that most of the values are between $-0.25 \mathrm{~m}$ and $+0.25 \mathrm{~m}$. This interval of values is related to the altimetry accuracy of the input point cloud. From Figure $3 a$, it can be noted that most of the pixels having values smaller than $-0.25 \mathrm{~m}$ are distributed on the building boundary because these points belong to building facades. That is why their heights are lower than those of the roof. If there are pixels having values smaller than $-0.25 \mathrm{~m}$ and they are situated far from the building boundary, these pixels are due to the noisy points. In conclusion, all roof pixels having values smaller than $-0.25 \mathrm{~m}$ are considered as noise.

Furthermore, the pixels surrounding the detectable details sometimes have values higher than $+0.25 \mathrm{~m}$ (inside the red circles in Figure $3 a$ ). This can be explained by the diameter of the laser spot, which generates planimetric error, in addition to the scanned points of vertical walls surrounding the detectable details. Therefore, the pixels surrounding the detectable details having values greater than $+0.25 \mathrm{~m}$ will be excluded. It is important to note that within the blue circles in Figure $3 a$, the pixels have considerable positive values. These pixels seem to present the missing roof details, as these details were not detected by the extended RANSAC algorithm. 
Thus, they appear newly in the error map matrix because they have a considerable altitude. Consequently, the missing roof details have high positive values in the error map matrix. In order to distinguish between the missing roof detail pixels and pixels having positive noise, a higher threshold than $+0.25 \mathrm{~m}$ is needed. From the histogram presented in Figure $3 b$ (inside the red circle), the missing roof details having values greater than $+0.50 \mathrm{~m}$ can be distinguished and detected. This also helps to avoid the false alarm, as the values between +0.25 and +0.50 sometimes cause a false alarm of missing roof details presence, while they are noisy points.

To be on the safe side, the pixels having values greater than $+0.50 \mathrm{~m}$ will be considered as missing roof details. For summarizing, the pixel values in error map matrix can be classified into six classes:

(1) Pixels having values belong to the interval $[-1,-0.25]$ present noise related to the facade's points.

(2) Pixels having values belong to the interval $[-0.25,+0.25]$ present most error map values; they belong to the principal roof planes.

(3) Pixels having values belong to the interval $[+0.25,+0.50]$ present noise.

(4) Pixels having values belong to the interval $[+0.50,+1]$ and surrounded the detectable roof details present noise.

(5) Pixels having values belong to the interval $[+0.50,+1]$ and do not situate nearby detectable roof details present missing roof details.

(6) Pixels belonging to detectable roof details planes will not be considered.

Applying these rules on the error map matrix allows the detection of the missing roof details. Figure $3 c$ presents the mask of missing roof details. Once this mask is calculated, it can be used 
in the next step for modelling of missing details.

\section{[Insert Figure 3 here]}

\section{Modelling of missing details}

Before speaking about the modelling task, it is important to note that there are two types of missing roof details on building roofs as is shown in Figure 4. The first type is the details having considerable altitudes and negligible area (see the red circle in Figure 4, its considerable altitudes can be noted regarding its long shadow in this image). The second type is the details having negligible altitudes (see blue circle in Figure 4, its negligible altitudes can be noted regarding its very short shadow in the image). The proposed approach allows only modelling the missing roof details having considerable altitudes. Since it depends on the height of missing roof details in the error map matrix, only the pixels having high height values, greater than $+0.5 \mathrm{~m}$, are considered.

\section{[Insert Figure 4 here]}

In order to detect the missing roof details, a region-growing algorithm is applied on the missing detail mask (Figure 3c). This procedure allows the independent detection of each missing roof detail. Regarding the negligible areas of these details, their geometric forms appear vague in the mask. Therefore, each detail will be presented by a square situated in the centre of gravity of each roof detail. Moreover, its area is equal to two pixels. In Figures $4 b$ and $6 d$, the $2 \mathrm{D}$ building models can be seen after considering the missing details, e.g., Figure $5 b$ presents 2D building model that has 12 missing details (in green). Figure $5 a$ presents the improved roof segmentation, and Figure $5 c$ shows the visualization of error map matrix after segmentation improvement (as it will be exposed in next section).

\section{[Insert Figure 5 here]}

After detection and modelling of the missing roof details, the second question arises in this paper 
about the enhancing of building roof segments.

\section{Improvement of building roof planes}

In the context of 3D building modelling, the more delicate step in data-driven approaches is the detection of building roof planes. Indeed, any misdetection of the roof planes will generate undesirable deformations in the 3D building model. The suggested improvement approach in this paper starts by the evolution of 2D roof segmentation result presented by the building label image. If the segmentation result is accepted, then the improvement operation can be carried out. Otherwise, the 3D building model will not be constructed until improving the roof segmentation result is accepted. In the case of acceptable segmentation, the building point cloud will be improved in order to decrease the noise quantity and, finally, it will be re-segmented to calculate the enhanced building label image. In this context, Lafarge and Mallet (2012), Li et al. (2019), and Shan and Toth (2018) suggested the use of a graph cut approach to optimize the delineation of roof elements initialized on a 2D supporting grid. However, the proposed approach in this paper differs from the existing papers. Indeed, the proposed approach goes further by using the enhanced building label image as the input for constructing the 2D building model before constructing the final 3D building model.

\subsection{Estimation of roof segmentation quality}

In Section 4, it is shown that the error map matrix can be used for visually estimating the accuracy of building roof segmentation. However, a numerical expression must be extracted from the error map matrix that expresses the accuracy and quality of the segmentation. For this purpose, a new accuracy factor is defined named SAQ (Segmentation Accuracy and Quality) factor. The SAQ factor presents the rate of the number of whole pixels in the error map matrix 
having values outside the interval $[-0.25,+0.25]$ to the total number of building pixels (See $S_{q}$ in Eq. (1)). That means the SAQ factor expresses the percentage of points having considerable noise.

$$
S_{q}=N / A \times 100,
$$

Where $S_{q}$ is the SAQ factor $(\%), N$ is the number of pixels outside the interval $[-0.25,+0.25]$ and $A$ is the total number of building pixels.

As the missing roof details were considered as undesirable noise, then it can be said that this factor expresses the number of missing detail points in addition to the points having noise. In fact, the number of points having considerable noise is very much larger than those representing the missing roof details. The values of this interval are assigned regarding the histogram of deviation distribution of the error map matrix for the case of a successful roof segmentation (Figure $3 b$ ). These values are related directly with the altimetry accuracy of the input point cloud, e.g., the altimetry accuracy of Strasbourg point cloud is less than that of Hermanni point cloud, then the interval is taken as $[-0.35,+0.35]$ for Strasbourg instead of $[-0.25,+0.25]$ for Hermanni and Vaihingen. More investigations have been carried out by monitoring and evaluating the error histograms of almost 100 building point clouds with different densities and urban typology. Then, the quality of the building roof segmentation according to the SAQ factor can be estimated according to Table 2 .

\section{[Insert Table 2 here]}

From Table 2, it can be noted that if the SAQ value is greater than $20 \%$, the result of building roof segmentation will be rejected. Otherwise, the SAQ factor allows evaluation of the successful level of the roof segmentation. 


\subsection{Enhancement of the roof segments}

It is noted that the points having deviations outside the interval $[-0.25,+0.25]$ generate a deformation in the plane equation. Therefore, these points are excluded after the first plane fitting, then the plane equation is re-estimated. This solution sometimes creates gaps among the plane area, and consequently, the distribution of points among the plane area will not be balanced. Moreover, the number of the excluded points is sometimes elevated.

From other viewpoint, a roof segmentation algorithm leads to the classification of the building point cloud into two classes, which are roof plane points and noisy points. If the segmentation is successfully achieved, then the noisy point class will contain only noisy points. Otherwise, if the segmentation is unsuccessful, then the noisy point class will contain both noisy points and roof plane points. Moreover, the roof plane point class will contain a mixture of roof plane points and noisy points. On the one hand, in the case of successful segmentation, if the noisy points are projected on the roof planes that will improve the quality of the point cloud because the number of noisy points is sharply decreased. Consequently, the re-application of the segmentation algorithm on the improved point cloud will provide an enhanced result. On the other hand, in the case of unsuccessful segmentation, if the points of the noisy point class are projected onto the detected roof planes that will not improve the quality of the point cloud because the point cloud in this case will suffer from deformation. Consequently, the re-application of the segmentation algorithm on the 'improved point' cloud will not provide an acceptable result.

A question arises: why are the noisy points projected and not omitted? If the number of points is changed by omitting the noisy points, the comparison between the results of the first and the second segmentation will not be obvious, considering that the suggested SAQ factor takes the number of points of noisy class into account. Moreover, the projection of the noisy point class in 
the case of unsuccessful segmentation deforms the roof geometry that leads to reject the segmentation result in the second segmentation.

In this context, a new solution is proposed for solving this problem. Once the SAQ factor is smaller than $20 \%$ (Table 2), in order to improve the building roof segmentation, every point having a noise value outside the interval $[-0.25,+0.25]$ must be projected on its corresponding plane. This operation will allow the generation of an improved and balanced point cloud. The next step is to re-segment the entirely improved point cloud of the building and re-calculate the SAQ factor. If the improvement of the building point cloud is valid, then the updated SAQ factor will be very small in comparison to the first one. Otherwise, if this improvement is not valid, then that will generate a high SAQ factor.

In conclusion, if the updated SAQ factor is smaller than the first one and smaller than $8 \%$ (see Section 8), then the generation of the improved point cloud as well as the roof improved segmentation result is acceptable. For example, Figure $5 a$ presents the re-segmentation of the building point cloud. Figure $5 b$ presents the improved 2D building model. In this example, the total number of building points is 4343 and the number of noisy points that are projected on roof planes is 331. Moreover, the SAQ factor before improvement is 5.32\% and its value after the improvement is $1.43 \%$. Tables 3 and 4 show the comparison between the two $2 \mathrm{D}$ building models before and after the improvement. It can be observed that the number of points of each principal plane varies before and after the improvement because the plane equations are enhanced. It can also be noted that the standard deviation value of each plane fitting becomes more accurate. Furthermore, this procedure will improve the quality of the 2D building model. This conclusion can be noted by comparing the improved building model in Figure $5 b$ with the building model without improvement in Figure $2 b$. 
On the other hand, if the value of the improved SAQ factor is greater than the first one and / or greater than $8 \%$ (see Section 8), then the generation of the improved point cloud as the roof segmentation result is unacceptable. Moreover, the first building segmentation is also invalid (this idea will be explored in more detail in the next section). Finally, if the value of the SAQ factor is smaller than $1 \%$, in this case it is not needed to improve the roof segmentation further because it is already excellent and the number of points having the noise is minimal. Moreover, when the value of the SAQ factor is smaller than $1 \%$, if the roof segmentation is improved, then it is possible to obtain the updated SAQ factor greater than the first one, but it stays in the same range. In this case, the segmentation will be correct because all these manoeuvres snip within the interval of an excellent case.

\subsection{Reason for invalidating a roof segmentation result}

Before explaining this idea, it must be remembered that the basic hypothesis used for modelling the missing roof details and for improving the roof segments is that the building roof is correctly segmented, and all its principal planes are correctly fitted. If this hypothesis is not realized, then the application of improvement has no meaning. In fact, the point cloud of one roof plane contains two classes of points: points having considerable noise, which are outside the interval [$0.25,+0.25]$ and points, which do not have considerable noise. The suggested improvement only targets the points having considerable noise. If the plane is mis-detected by the roof segmentation algorithm, the improvement will also be applied to points without considerable noise and that is not acceptable. Thus, it will give an unexpected result. Let us take the example presented in Figure 6b, which presents a mis-segmented roof. The value of the SAQ factor before improvement is equal to $22.51 \%$ but its value after improvement is equal to $25.52 \%$. As a result, the SAQ value after improvement does not realize successfully the supposed conditions. 
This leads to rejection of the improved as well as the first segmentation results. Hence, the influence of mis-segmentation will appear in the form of deformations in the 2D and 3D building models. At this stage, several modification trails of segmentation algorithm parameters are tested. Then, a better result in visually level is considered (Figure 6c). However, the architectural complexity of the roof in addition to a weak point density and altimetry accuracy are the reasons for getting considerable deviations in the new error map matrix after enhancing the parameters of the roof segmentation algorithm (Figure $6 d$ ). Moreover, the SAQ factor is still high $\left(S_{q}=\right.$ 26.40\%). Thus, in the case of the building presented in Figure 6, several reasons contribute to the segmentation failure. Despite attempting to exclude one of these reasons, which is the selection of segmentation parameter values, the segmentation result is still unacceptable, because several couples or triples of neighbouring planes are merged together. This is explained by the fact that one plane may be detected instead of two when there is a low height difference between two planes and the step edge or the narrow plane between them is not detected separately. The same can also happen when two intersecting neighbouring planes have almost the flat slopes.

Moreover, both plane areas and point density increase the scene complexity. Consequently, the error map matrix (Figure $6 d$ ) presents the unacceptable quantity of error made in the segmentation after modification of the segmentation parameters.

\section{[Insert Figure 6 here]}

[Insert Table 3 here]

\section{[Insert Table 4 here]}

In Figure 7, the building roof contains 86 missing details and about 80 of these details are modelled correctly (Figure $7 d$ ). Furthermore, it can be observed from the error map matrix (Figure $7 b$ ) that the building roof is successfully segmented (Figure $7 b$ ) where the missing details are successfully modelled and the roof segments are successfully improved. The value of SAQ 
factor before the improvement is $12.31 \%$ and after the improvement is $6.99 \%$.

Before finishing, it is necessary to observe that after enhancing the roof plane equations, it is possible that other new points will have considerable noise (Figure $5 c$ ). Nevertheless, the number of these points has to be negligible or less than that of before enhancing. That is why the new SAQ factor is not equal to zero. If their number is high, then the SAQ factor value will be also high and, consequently, the roof segmentation and the improvement will be rejected.

[Insert Figure 7 here]

\section{8. $\quad$ Parameter setting}

There are limited parameters used by the proposed approach. One of the parameter values is selected from the existing literature and the others are chosen by the experiments.

The first employed parameter is the deviation interval value in error map matrix. In Section 4, it has been shown that in order to visualize the error map matrix, the deviations have to be clipped to be within a given interval. For this purpose, Tarsha Kurdi (2008) and Dorninger and Pfeifer (2008) suggest the employment of this interval $[-1 \mathrm{~m},+1 \mathrm{~m}]$. That is why the same interval value is used in this research.

The second parameter is the SAQ factor value that describes the quality of building roof segmentation (Table 2). Moreover, the last parameter is the successful conditions of the resegmentation of a building point cloud according to the SAQ factor value which are:

- The SAQ factor value after improvement has to be smaller than the SAQ factor value before improvement.

- The SAQ factor value after improvement has to be smaller than $8 \%$. 
The Table 2 values in addition to the last two conditions have been assigned through monitoring and evaluating the error histograms of almost 100 building point clouds with variant densities and variant urban typology.

\section{Relationship between missing roof details and SAQ factor}

As the SAQ factor expresses the roof segmentation accuracy and it summarizes the error map matrix content, then it is necessary to take its value into account before modelling the missing roof details. When the value of the SAQ factor refers to the success of building roof segmentation (Table 2), only in this case the procedure of missing detail modelling can be carried out. Otherwise, the missing details cannot be modelled because the roof segmentation is not achieved successfully. In the case of successful segmentation result, another control of improvement validity will be achieved when a new error map matrix is re-calculated after improvement. The organigram presented in Figure 1 exposes the consecutive steps of the proposed approach. It highlights the two control steps which validate the modelling of missing roof details in addition to the improvement of roof segments. This organigram shows the relationship between the main three operations suggested in this paper which are: modelling of

missing roof details, accuracy evaluation by calculating the SAQ factor and the improvement of building roof segments. Consequently, it is now understandable why these three operations are presented together in this paper. At this stage, it is inevitable to discuss the advantages and the disadvantages of the proposed approach.

\section{Advantages and disadvantages}

The calculation of the SAQ factor provides an important index about the roof segmentation quality. Indeed, this quality estimation is carried out into two stages. The first one is before 
improvement, where the SAQ factor must be calculated for evaluating the improvement possibility. This factor, in addition to the number of missing roof details, provides an accurate index about the segmentation quality. In the next stage, the new value of the SAQ factor after improvement also gives an accurate index about the roof segmentation quality, e.g., if the SAQ value after the improvement is near to zero, it means the roof segmentation is almost perfect, but if this value is greater than $8 \%$ that means the roof segmentation is rejected (see Section 8). Secondly, the roof segments improvement enhance the accuracy of roof plane equations, as it reduces the noise and drives to refine the plane equations. This can be clearly seen through the standard deviation values of the roof planes after improvement (Tables 3 and 4). Finally, the segments improvement provides an opportunity to enhance the inner roof planes boundaries due to the refining of roof plane equations. Thus, the $2 \mathrm{D}$ building model in addition to the $3 \mathrm{D}$ building model will also be enhanced. The importance of the accuracy estimation (SAQ factor) comes from getting error alarm before starting 3D building modelling step, because the roof missegmentation will generate a considerable deformation in 3D building model.

At this stage, it is important to discuss about the disadvantages of point cloud improvement. The idea of improvement of building point cloud is dangerous because any error during this operation may lead to deformation of the roof geometry. But the link of improvement procedure to the value of the SAQ factor makes its application secure, as the SAQ factor before and after improvement gives an evaluation and control of roof segmentation quality, which prevents retouching the points that have no noise.

\section{Results}

Two test sites in the Vaihingen point cloud and the Hermanni point cloud have been selected. 
These three test sites contain 21, 6 and 12 buildings, respectively. Figures 8,9 and 10 present the final result of automatic 2D building modelling for these test sites. Moreover, three colours are used, the blue represents the building outer boundaries, the red represents the inner roof plane boundaries and the green represents the missing roof details. Three buildings are zoomed in Hermanni site (Figure $8 c$ ) for illustrating the model deformations and the missing roof details.

\section{[Insert Figure 8 here] \\ [Insert Figure 9 here]}

Finally, the quantitative analysis and the accuracy of these results are carried out in the following section.

\section{[Insert Figure 10 here]}

\section{Accuracy and analysis}

Ostrowski et al. (2018) and Cheng et al. (2015) summarize the accuracy estimation approaches of the literature into two classes. In the first approach class, which is called feature-based, a reference model is required in order to compare the calculated model with it. In contrast, the approaches in the second class, which are called Lidar-based, consider the Lidar point cloud as reference data. However, this classification is very brief because the methods of construction of the reference models in the first class are not taken into account. In the context of Lidar-based approaches, it is suggested that the Lidar point cloud is used as reference data Tarsha Kurdi (2008), Dorninger and Pfeifer (2008), Ostrowski et al. (2018), Erberink and Vossleman (2011), Akca et al. (2010), Sampath and Shan (2010) and Park et al. (2019). Consequently, one of the crucial elements of their approaches of modelling accuracy estimation is the calculation of distances (point per point) between the 3D building model and the point cloud. Hence, the accuracy estimation approach employed in this paper belongs to the second class where the 
building point cloud is considered as reference data.

In order to estimate the accuracy of the $2 \mathrm{D}$ building models presented in Figures $8 b, 9 b$ and $10 b$, the SAQ factors are calculated before and after the improvement (see Tables 5, 6 and 7). In Table 5, it can be noted that all buildings have an excellent accuracy estimation regarding the value of SAQ factor after improvement (SAQ is smaller than 5\%, see Table 2). These results refer to the success of building roof segmentation and to validity of modelling of missing details in addition to the validity of roof segments improvement.

In Table 6, Buildings 3, 8, 12, 13, 16, 17, 18, 20 and 21 have very accurate results after the first roof segmentation because their SAQ factors are smaller than 1\% (see Table 2). Then, they don't need to improve their roof segmentation. Therefore, the SAQ factors after improvement are not calculated again. In Table 7, there is not any rejected result and the six buildings have an excellent segmentation accuracy. Furthermore, three building segments don't need improvement (Buildings 1, 5 and 6) because their SAQ factor values after the first segmentation are smaller than 1\%. Tables 5 and 6 show that the obtained results in Vaihingen point cloud are valid. Furthermore, the modelling of missing roof details are also valid.

\section{[Insert Table 5 here]}

[Insert Table 6 here]

\section{[Insert Table 7 here]}

In order to estimate the functionality of the suggested approach for automatic modelling of missing roof details, the Missing Roof Details (MRD) are counted on the aerial image of Vaihingen test sites (Tables 8 and 9). For achieving this goal, it is observed that the missing details have considerable shadows in the areal image because they have considerable altitude values. Then, this number is compared to the Number of Missing Roof Details which are 
Modelled (Number of MMRD). Tables 8 and 9 expose this comparison where two cases are envisaged:

(1) Number of MRD = Number of MMRD in 23 buildings among 27 buildings. This result confirms the efficiency of the proposed approach.

(2) Number of MRD < Number of MMRD in 4 buildings. The major difference between the two numbers is equal to 2 in buildings number 4 and 7 (Table 8). That can be explained by the presence of noise as an obstacle on the roof or in the air over the building or any other noise type that generates noise points which considered as missing roof detail.

It is also possible to meet the case of absence of missing roof detail as the case of building presented in Figure 7, where the number of missing details is equal to 86 and the number of modelled missing details is equal to 80 . The interpretation of this case is by the absence of scanning points that cover the roof details, or the height of missing detail is smaller than $0.5 \mathrm{~m}$.

At this stage, it can be noted that the obtained results are satisfactory on the level of building segments improvement and on the level of modelling of the missing roof details.

[Insert Table 8 here]

[Insert Table 9 here]

Another way of accuracy estimation is applied by calculating the standard deviation of each 2D building model. For this purpose, the building pixel values in error map matrix are used (without clipping them within the interval $[-1 \mathrm{~m},+1 \mathrm{~m}]$ ) because these pixel values present the deviations between the point cloud and the roof fitted planes. Figure 11 exposes the standard deviations of 2D building models presented in Figures 8, 9 and 10, where 27 building models of Vaihingen site are compared to 12 building models in Hermanni site. It shows that the models of Vaihingen 
site are more accurate than those of Hermanni site; despite the flight height of Hermanni data (200 $\mathrm{m}$ ) being smaller than that of Vaihingen data $(500 \mathrm{~m})$. The reason for this accuracy difference comes from the quality of Lidar sensor, which is related to the date of scanning (see Table 1). However, the modelling accuracy of the data sets are smaller than $0.2 \mathrm{~m}$, which can be considered as excellent results regarding the employed point cloud accuracy.

\section{[Insert Figure 11 here]}

Finally, sometimes it is observed that the building model contains some deformations despite its accuracy indexes being of high quality, e.g., the outer boundary of Building 9 in Figure $9 b$ suffers a deformation. The reason for the deformation in this example are the presence of a tree nearby the building, which covers small part of it. In this case, the building point cloud is deformed but the building model is faithful to building point cloud. Hence, there are three ordered models of the each building: the original building, the point cloud and the calculated model. The calculated model has to be faithful to the building point cloud. On the other hand, it necessary to estimate how much the point cloud is faithful to the original building. Then, more investigations are required in order to answer this question.

\section{Conclusion}

In this paper, the suggested approach focuses on analyzing the error map matrix in the context of automatic modelling of missing roof details. The missing roof details have negligible areas in addition to considerable altitudes. At this stage, the error map matrix is used for several purposes. The main goal is to estimate visually and numerically the accuracy of building roof segmentation. Hence, the visualization of the error map allows the visual estimation of the segmentation accuracy, whereas the SAQ factor serves to evaluate the accuracy numerically. The second application of the error map matrix is to detect and to model the missing roof details. If 
the building roof is successfully segmented, then the missing roof details can be extracted starting from the error map matrix and, consequently, they can be modelled. At this stage, it is important to underline that the point density plays an important role in the success of missing details modelling regarding the small areas of these details. Moreover, the success of roof segmentation is related to several factors, such as the successful selection of the roof segmentation algorithm parameters, the harmonization between the roof geometry and the adopted hypotheses by segmentation algorithm, and the point cloud characteristics, such as point density, accuracy, regularity, homogeneity and noise quantity. Consequently, the point cloud density value plays a crucial role in the success of the roof segmentation as well as the roof missing detail modelling. The third application of the error map matrix is the improvement of building roof segments by enhancing the building point cloud and then re-segmenting the building roof. In the case of roof mis-segmentation, more investigations are required for finding the main reasons for the segmentation failure and the suggestions for the suitable solutions. Finally, the modelling accuracy estimation allows to measure the fidelity of the calculated model to the building point cloud. However, more investigations are required in order to estimate the fidelity of building point cloud to the original building. Once the building roof is segmented successfully, the way will be paved toward constructing automatically and accurately the 3D building model starting from Lidar data.

Acknowledgement: The Vaihingen data set was provided by the German Society for Photogrammetry, Remote Sensing and Geoinformation (DGPF) (Cramer, 2010): http://www.ifp.unistuttgart.de/dgpf/DKEP-Allg.html. 


\section{References:}

Acar, H. Karsli, F. Ozturk, M., and M. Dihkan. 2019. “Automatic detection of building roofs from point clouds produced by the dense image matching technique”. International Journal of Remote Sensing, Vol. 40, No. 1, 138-155, https://doi.org/10.1080/01431161.2018.1508915.

Akca, D. Freeman, M. Sargent, I., and A. Gruen. 2010. "Quality assessment of 3D building data." The Photogrammetric Record, 25(132), pp.339-355.

Alharthy, A., and J.S. Bethel. 2004. "Detailed building reconstruction from airborne laser data using a moving surface method.” Int. Arch. of Photogrammetry and Remote Sensing, Vol. XXXV, Part B3, 6p.

Awrangjeb, M. Gilani, S.A.N., and F.U. Siddiqui. 2018. "An effective data-driven method for 3D building roof reconstruction and robust change detection." Remote Sens. 2018, 10, 1512; doi: $10.3390 / \mathrm{rs} 10101512$.

Brenner, C. 2000. “Towards fully automatic generation of city models.” Int. Arch. of Photogrammetry and Remote Sensing, vol. XXXIII, Part 3. Amsterdam, pp. 85-92.

Bretar, F., and M. Roux. 2005. "Hybrid image segmentation using Lidar 3D planar primitives." Int. Arch. of Photogrammetry and Remote Sensing, vol. XXXVI, Part 3/W19 pp.72-78.

Bulatov, D., and M. Pohl. 2013. "Detection of small roof details in image sequences.” Kämäräinen JK., Koskela M. (eds) Image Analysis. SCIA 2013. Lecture Notes in Computer Science, vol 7944. Springer, Berlin, Heidelberg.

Cao, R. Zhang, Y. Liu, X., and Z. ZhaoTo. 2017. “3D building roof reconstruction from airborne Lidar point clouds: a framework based on a spatial database." International journal of geographical information science, 2017, VOL. 31, NO. 7, 1359-1380. DOI: 10.1080/13658816.2017.1301456.

Cheng, L. Zhang, W. Zhong, L. Du, P., and M. Li. 2015. "Framework for evaluating visual and geometric quality of three-dimensional models." IEEE journal of selected topics in applied earth observations and remote sensing, vol. 8, no. 3, March 2015, DIO: 10.1109/JSTARS.2014.2370753.

Cramer, M. 2010. "The DGPF test on digital aerial camera evaluation - overview and test design." Photogrammetrie - Fernerkundung - Geoinformation 2(2010):73-82.

Demir, N. 2018. “Automated detection of 3D roof planes from Lidar data." Journal of the Indian Society of Remote Sensing. (August 2018) 46(8):1265-1272. https://doi.org/10.1007/s12524-018-0802-2.

Douglas, D.H., and T.K. Peucker. 1973. "Algorithms for the reduction of the number of points required to represent a digitized line or its caricature.” The Canadian Cartographer, 10 (2), 112 -122. 1973. 
Dorninger, P., and N. Pfeifer. 2008. "A comprehensive automated 3D approach for building extraction, reconstruction, and regularization from airborne laser scanning point clouds." Sensors, 8(11), pp.7323-7343.

Elaksher, A. F., and J. S. Bethel. 2002. "Reconstructing 3D buildings from Lidar data." Int. Arch. of Photogrammetry and Remote Sensing, Vol. XXXIV, part 3A/B, pp. 102-107.

Elberink, S.O., and G. Vosselman. 2011. "Quality analysis on 3D building models reconstructed from airborne laser scanning data." ISPRS Journal of Photogrammetry and Remote Sensing, 66(2), pp.157-165. http://doi.org/10.1016/j.isprsjprs.2010.09.009.

Forlani, G. Nardinocchi, C. Scaioni, M., and P. Zingaretti. 2006. "Complete classification of raw Lidar data and 3D reconstruction of buildings." Pattern Anal. Applic. (2006) 8. DOI 10.1007/s10044005-0018-2, pp. 357-374.

Ghaffarian, S. Ghaffarian, S. Elmerabet, Y. Samir, Z., and Y. Ruicheck. 2016. "Automatic building roof segmentation based on PFICA algorithm and morphological filtering from Lidar point clouds." In 37th Asian Conference on Remote Sensing (ACRS 2016), promoting spatial data infrastructure for sustainable economic development, 17-21 October 2016, Colombo, Sri Lanka.

Hofmann, A. D. 2004. "Analysis of tin-structure parameter spaces in airborne laser scanner data for 3D building model generation." Int. Archives of Photogrammetry and Remote Sensing, Vol. XXXV, part B3, 6p.

Kadaa, M., and L. McKinley. 2009. “3D building reconstruction from Lidar based on a cell decomposition approach.” IAPRS, Vol. XXXVIII, Part 3/W4, Paris, France, 3-4 September, 2009.

Lafarge, F., and C. Mallet. 2012. "Creating large-scale city models from 3D point clouds: a robust approach with hybrid representation.” Int. J. Comput. Vision 99 (1), 69-85.

Li, M. Rottensteiner, F., and C. Heipke. 2019. "Modelling of buildings from aerial Lidar point clouds using TINs and label maps.” ISPRS Journal of Photogrammetry and Remote Sensing 154 (2019) 127-138. https://doi.org/10.1016/j.isprsjprs.2019.06.003.

Oda, K. Takano, T. Doihara, T., and R. Shibasaki. 2004. "Automatic building extraction and 3D city modelling from Lidar data based on Hough transformation." Int. Arch. of Photogrammetry and Remote Sensing, Vol. XXXV, part B3, pp.16-23.

Ostrowski, W. Pilarska, M. Charyton, J., and K. Bakuła. 2018. "Analysis of 3D building models accuracy based on the airborne Laser scanning point clouds." Int. Arch.of the Photogrammetry, Remote Sensing and Spatial Information Sciences, Volume XLII-2, 2018, ISPRS TC II Mid-term Symposium “Towards Photogrammetry 2020”, 4-7 June 2018, Riva del Garda, Italy. https://doi.org/10.5194/isprs-archives-XLII-2-797-2018. 
Overby, J. Bodum, L. Kjems, E., and P. M. Ilsoe. 2004. “Automatic 3D building reconstruction from airborne laser scanning and cadastral scanning and cadastral data using Hough transform." Int. Arch. of Photogrammetry and Remote Sensing, Vol. XXXV, part B3, 6p.

Park, J. Lee, I. Choi, Y., and Y.J. Lee. 2006. "Automatic extraction of large complex buildings using Lidar data and digital maps." Workshop ISPRS. Com III, Photogrammetric Computer Vision PCV Bonn, Germany 20 - 22 September 2006.

Park, S.Y. Lee, D.G. Yoo, E.J., and D.C. Lee. 2019. "Segmentation of Lidar data using multilevel cube code.” Journal of Sensors, Volume 2019, Article ID 4098413, 18 pages, https://doi.org/10.1155/2019/4098413.

Rabbani, T., and F. Van den Heuvel. 2005. "Efficient Hough transform for automatic detection of cylinders in point clouds.” Int. Arch. of Photogrammetry and Remote Sensing, vol XXXVI, part 3/W19, pp. 60-65.

Rottensteiner, F. 2003. “Automatic generation of high-quality building models from Lidar data.” IEEE $C G \& A 23(6)$, pp. $42-51$.

Rottensteiner, F. 2010. "Roof plane segmentation by combining multiple images and point clouds." Int. Arch. of Photogrammetry Remote Sensing and Spatial Sciences, 38(3A), 245-250.

Salehi, A., and A. Mohammadzadeh. 2017. "Building roof reconstruction based on residue anomaly analysis and shape descriptors from Lidar and optical data." Photogrammetric Engineering \& Remote Sensing, 83(4), 281-291.

Sampath, A., and J. Shan. 2006. "Clustering based planar roof extraction from Lidar data." American Society for Photogrammetry and Remote Sensing, Annual Conference, Reno, Nevada, May 1-6.

Sampath, A., and J. Shan. 2010. "Segmentation and reconstruction of polyhedral building roofs from aerial Lidar point clouds." IEEE transactions on geoscience and remote sensing, vol. 48, no. 3, march 2010.

Schenk, T., and B. Csatho. 2002. "Fusion of Lidar data and aerial imagery for a more complete surface description.” Int. Arch. of the Photogrammetry, Remote Sensing and Spatial Information Sciences, Proceedings of the ISPRS Commission III Symposium on Photogrammetric and Computer Vision, Volume XXXIV, Graz, Austria, 2002, pp. 310-317.

Shan, J., and C.K. Toth. 2018. Topographic laser ranging and scanning principles and processing. Second edition, by Taylor \& Francis Group, LLC. ISBN- 13: 978-1-4987-7227-3 (hardcover), 630 P, 2018.

Sohn, G., and I. Dowman. 2007. "Data fusion of high-resolution satellite imagery and Lidar data for automatic building extraction." ISPRS Journal of Photogrammetry and Remote Sensing, 62(1), May, 43-63. 
Sohn, G. Huang, X., and V. Tao. 2008. "Using a binary space partitioning tree for reconstructing polyhedral building models from airborne Lidar data." Photogrammetric Engineering and Remote Sensing, 1436 November 2008.

Tarsha Kurdi, F. 2008. “3D building extraction and modelling from Lidar data.” $\mathrm{PhD}$ grade at Photogrammetry and Geomatics Group MAP-PAGE UMR 694, Graduate School of Science and Technology of Strasbourg (INSA of Strasbourg) (ULP University).

Tarsha Kurdi, F. Awrangjeb, M., and N. Munir. 2019. “Automatic 2D modelling of inner roof planes boundaries starting from Lidar data." 14th 3D GeoInfo 2019, 26-27 September, Singapore, DOI: 10.5194/isprs-annals-IV-4-W8-107-2019.

Tarsha Kurdi, F. Landes, T., and P. Grussenmeyer. 2008. "Extended RANSAC algorithm for automatic detection of building roof planes from Lidar data." The Photogrammetric Journal of Finland, vol. $21, \mathrm{n}^{\circ} 1,2008$, pp. 97-109.

Tarsha Kurdi, F. Landes, T., and P. Grussenmeyer. 2007. "Hough-transform and extended RANSAC algorithms for automatic detection of 3D building roof planes from Lidar data.” ISPRS Workshop on Laser Scanning 2007 and SilviLaser 2007, Espoo, Finland, Sept. 12-14th. ISPRS, Int. Arch. of Photogrammetry, Remote Sensing and Spatial Information Systems. Vol. XXXVI, Part 3 / W52, 2007, pp. 407-412.

Vosselman, G., and S. Dijkman. 2001. “3D building model reconstruction from point clouds and ground plans." Int. Arch. of the Photogrammetry, Remote Sensing, XXXIV-3/W4, pp. 37-43.

Wen, X. Xie, H. Liu, H., and L. Yan. 2019. “Accurate reconstruction of the lod3 building model by integrating multi-source point clouds and oblique remote sensing imagery." ISPRS Int. J. Geo-Inf. 2019, 8, 135; doi: 10.3390 /ijgi8030135.

Xiao, Y. Wang, C. Li, J. Zhang, W. Xi, X. Wang, C., and P. Dong. 2015. "Building segmentation and modelling from airborne Lidar data." International Journal of Digital Earth, 8:9, 694-709, DOI: 10.1080/17538947.2014.914252.

Xiong, B. Jancosek, M. Oude Elberink, S., and G. Vosselman. 2015. "Flexible building primitives for 3D building modelling." ISPRS Journal of Photogrammetry and Remote Sensing 101 (2015) 275290.

Zhang, W. Wang, H. Chen, Y. Yan, K., and M. Chen. 2014. "3D Building roof modelling by optimizing primitive's parameters using constraints from Lidar data and aerial imagery." Remote Sens. 2014, 6, 8107-8133; doi: 10.3390/rs6098107.

Zheng, Y. Weng, Q., and Y. Zheng. 2017. “A hybrid approach for three-dimensional building reconstruction in Indianapolis from Lidar data.” Remote sens. 2017, 9, 310; doi: $10.3390 / \mathrm{rs} 9040310$. 
Table 1. Characteristics of the three datasets used in this paper

\begin{tabular}{|c|c|c|c|}
\hline Acquisition & $\begin{array}{c}\text { End of June } \\
2002\end{array}$ & $\begin{array}{c}\text { Begin September } \\
2004\end{array}$ & 21 August 2008 \\
\hline Sensor & TopoEye & $\begin{array}{c}\text { TopScan (Optech } \\
\text { ALTM 1225) }\end{array}$ & $\begin{array}{c}\text { Leica Geosystems Leica } \\
\text { ALS50 system }\end{array}$ \\
\hline $\begin{array}{c}\text { Points density } \\
\text { (points } \text { m }^{-2}\end{array}$ & $7-9$ & 1.3 & $6.7-4$ \\
\hline $\begin{array}{c}\text { Flight height } \\
(\mathrm{m})\end{array}$ & 200 & 1440 & 500 \\
\hline
\end{tabular}

Table 2. Quality of building roof segmentation according to SAQ factor

\begin{tabular}{|l|l|l|l|l|l|}
\hline$S A Q(\%)$ & $>20$ & {$[20,15]$} & {$[10,15]$} & {$[5,10]$} & {$[0,5]$} \\
\hline $\begin{array}{l}\text { Accuracy } \\
\text { estimation }\end{array}$ & Rejected & Passable & Good & Very good & Excellent \\
\hline
\end{tabular}

Table 3. Building model before improvement

\begin{tabular}{|c|c|c|c|}
\hline $\begin{array}{c}\text { Plane } \\
\text { number }\end{array}$ & $\begin{array}{c}\text { Number } \\
\text { of points }\end{array}$ & $\begin{array}{c}\text { Standard } \\
\text { deviation }(\mathrm{m})\end{array}$ & \\
\hline 1 & 1119 & 0.62 & \multirow{2}{*}{$\begin{array}{c}\text { Principal } \\
\text { roof planes }\end{array}$} \\
\hline 2 & 1029 & 0.50 & \\
\hline 3 & 1095 & 0.18 & \\
\hline 4 & 980 & 0.43 & \\
\hline 5 & 25 & 2.25 & Detectable \\
\hline 6 & 19 & 1.11 & roof details \\
\hline 7 & 61 & 0.56 & \\
\hline 8 & 15 & 1.55 & \\
\hline
\end{tabular}


Table 4. Building model after improvement

\begin{tabular}{|c|c|c|c|}
\hline $\begin{array}{c}\text { Plane } \\
\text { number }\end{array}$ & $\begin{array}{c}\text { Number } \\
\text { of points }\end{array}$ & $\begin{array}{c}\text { Standard } \\
\text { deviation }(\mathrm{m})\end{array}$ & \\
\hline 1 & 1115 & 0.16 & \multirow{2}{*}{$\begin{array}{c}\text { Principal } \\
\text { roof planes }\end{array}$} \\
\hline 2 & 1044 & 0.14 & \\
\hline 3 & 1033 & 0.11 & \\
\hline 4 & 1042 & 0.13 & \\
\hline 5 & 25 & 2.24 & \multirow{2}{*}{ Detectable } \\
& roof details \\
\hline 6 & 19 & 1.11 & \\
\hline 7 & 50 & 0.55 & \\
\hline 8 & 15 & 1.54 & \\
\hline
\end{tabular}

Table 5. The values of SAQ factor before and after improvement of Hermanni site

\begin{tabular}{|c|c|c|c|c|c|c|}
\hline $\begin{array}{c}\text { Building } \\
\text { number }\end{array}$ & 1 & 2 & 3 & 4 & 5 & 6 \\
\hline SAQ1 (\%) & 8.46 & 2.79 & 4.76 & 5.55 & 6.24 & 8.23 \\
\hline SAQ2 (\%) & 0.94 & 0.92 & 2.03 & 1.64 & 1.55 & 3.11 \\
\hline $\begin{array}{c}\text { Building } \\
\text { number }\end{array}$ & 7 & 8 & 9 & 10 & 11 & \\
\hline SAQ1 (\%) & 2.15 & 2.77 & 2.30 & 2.85 & 3.50 & \\
\hline SAQ2 (\%) & 0.35 & 0.35 & 1.37 & 0.70 & 0.87 & \\
\hline
\end{tabular}

Table 6. The values of SAQ factor before and after improvement of first Vaihingen site

\begin{tabular}{|c|c|c|c|c|c|c|c|}
\hline $\begin{array}{c}\text { Building } \\
\text { number }\end{array}$ & 1 & 2 & 3 & 4 & 5 & 6 & 7 \\
\hline SAQ1 (\%) & 1.36 & 2.15 & 0.14 & 3.53 & 9.59 & 2.55 & 1.22 \\
\hline SAQ2 (\%) & 0.86 & 0.90 & -- & 1.44 & 1.17 & 0.84 & 0.25 \\
\hline $\begin{array}{c}\text { Building } \\
\text { number }\end{array}$ & 8 & 9 & 10 & 11 & 12 & 13 & 14 \\
\hline SAQ1 (\%) & 0.16 & 1.54 & 1.71 & 6.4 & 0.92 & 0.94 & 2.64 \\
\hline SAQ2 (\%) & -- & 0 & 1.65 & 2.37 & -- & -- & 1.00 \\
\hline $\begin{array}{c}\text { Building } \\
\text { number }\end{array}$ & 15 & 16 & 17 & 18 & 19 & 20 & 21 \\
\hline SAQ1 (\%) & 2.61 & 0.26 & 0.43 & 0.38 & 1.73 & 0 & 0,98 \\
\hline SAQ2 (\%) & 0.83 & -- & -- & -- & 0.44 & -- & -- \\
\hline
\end{tabular}


Table 7. The values of SAQ factor before and after improvement of second Vaihingen site

\begin{tabular}{|c|c|c|c|c|c|c|}
\hline $\begin{array}{c}\text { Building } \\
\text { number }\end{array}$ & 1 & 2 & 3 & 4 & 5 & 6 \\
\hline SAQ1 (\%) & 0.82 & 1.30 & 1.16 & 1.70 & 0.58 & 0.15 \\
\hline SAQ2 (\%) & -- & 0.12 & 0.06 & 0.10 & -- & -- \\
\hline
\end{tabular}

Table 8. Comparison of the correct number of missing roof details and the number of modelled missing roof details; first Vaihingen site (MRD: Missing Roof Details; MMRD: Modelled Missing Roof Details)

\begin{tabular}{|c|c|c|c|c|c|c|c|}
\hline $\begin{array}{c}\text { Building } \\
\text { number }\end{array}$ & 1 & 2 & 3 & 4 & 5 & 6 & 7 \\
\hline $\begin{array}{c}\text { Number } \\
\text { of MRD }\end{array}$ & 0 & 0 & 0 & 3 & 3 & 6 & 2 \\
\hline $\begin{array}{c}\text { Number } \\
\text { of MMRD }\end{array}$ & 0 & 0 & 0 & 5 & 3 & 6 & 2 \\
\hline $\begin{array}{c}\text { Building } \\
\text { number }\end{array}$ & 8 & 9 & 10 & 11 & 12 & 13 & 14 \\
\hline $\begin{array}{c}\text { Number } \\
\text { of MRD }\end{array}$ & 0 & 0 & 0 & 0 & 0 & 3 & 1 \\
\hline $\begin{array}{c}\text { Number } \\
\text { of MMRD }\end{array}$ & 0 & 0 & 0 & 0 & 0 & 3 & 1 \\
\hline $\begin{array}{c}\text { Building } \\
\text { number }\end{array}$ & 15 & 16 & 17 & 18 & 19 & 20 & 21 \\
\hline $\begin{array}{c}\text { Number } \\
\text { of MRD }\end{array}$ & 7 & 0 & 1 & 0 & 1 & 0 & 1 \\
\hline $\begin{array}{c}\text { Number } \\
\text { of MMRD }\end{array}$ & 9 & 1 & 1 & 0 & 1 & 0 & 1 \\
\hline
\end{tabular}

Table 9. Comparison of the correct number of missing roof details and the number of modelled missing roof details; second Vaihingen site (MRD: Missing Roof Details; MMRD: Modelled Missing Roof Details)

\begin{tabular}{|c|l|l|l|l|l|l|}
\hline $\begin{array}{c}\text { Building } \\
\text { number }\end{array}$ & 1 & 2 & 3 & 4 & 5 & 6 \\
\hline $\begin{array}{c}\text { Number of } \\
\text { MRD }\end{array}$ & 5 & 7 & 2 & 5 & 4 & 1 \\
\hline $\begin{array}{c}\text { Number of } \\
\text { MMRD }\end{array}$ & 5 & 7 & 3 & 5 & 4 & 1 \\
\hline
\end{tabular}




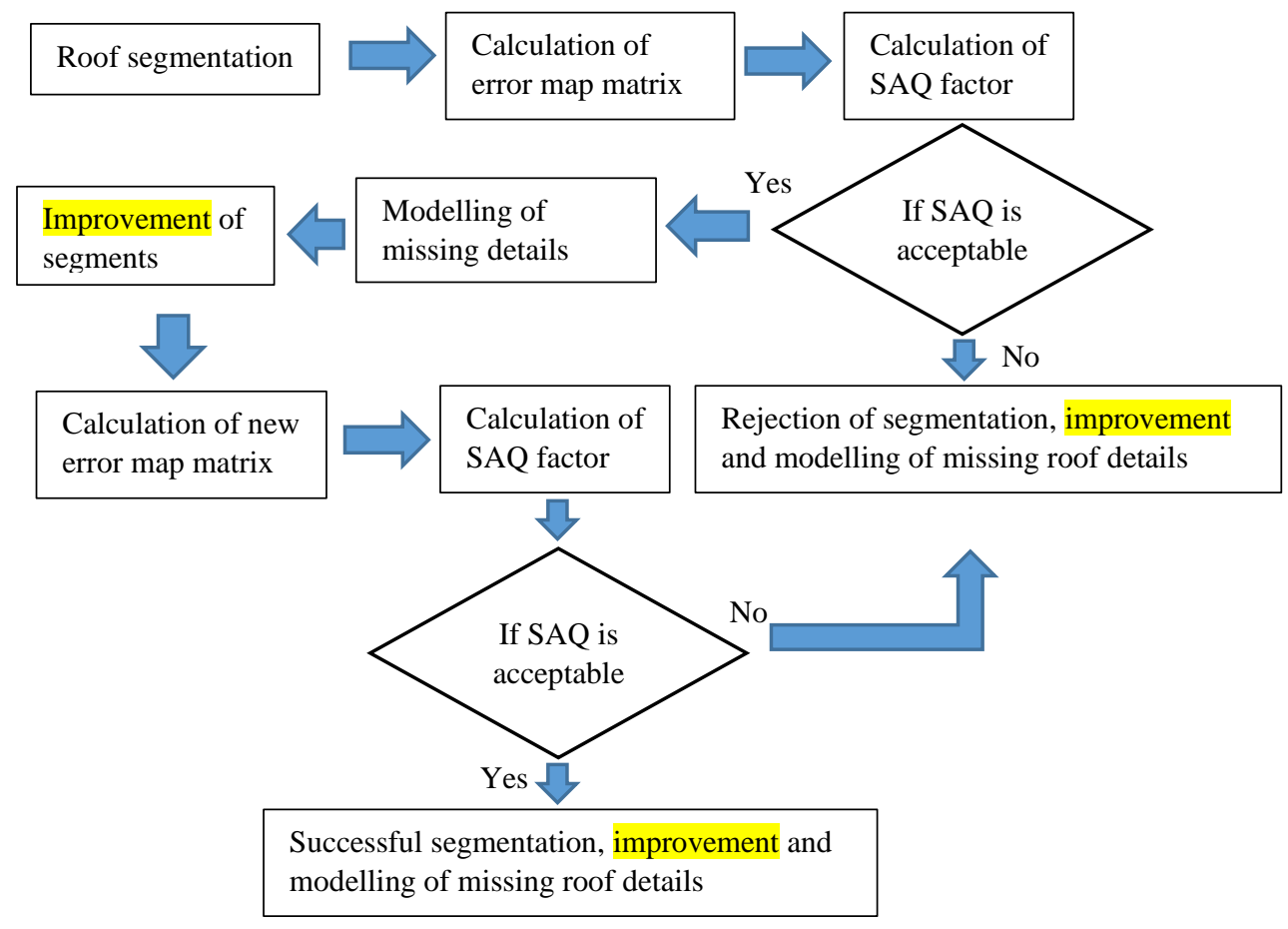

Figure1. Organigram of consecutive steps of the suggested approach



(a)

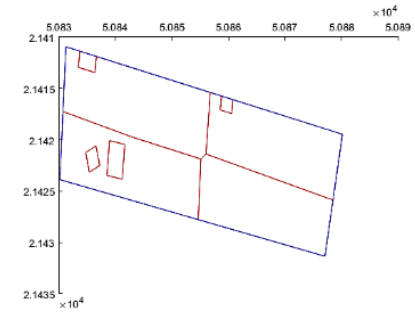

(b)

Figure 2. Segmentation of the building point cloud (Hermanni data set): (a) Building label image (the colours present the plane numbers); (b) 2D building model (blue lines present outer building boundary and red lines present the inner roof boundaries) 


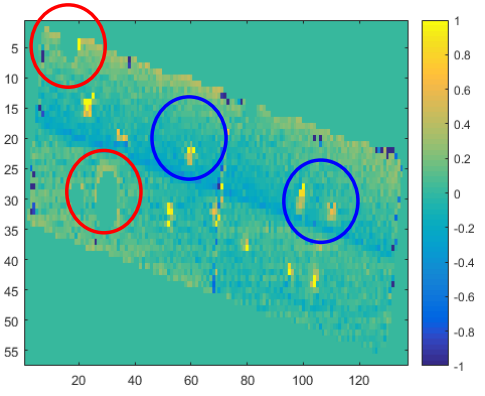

(a)

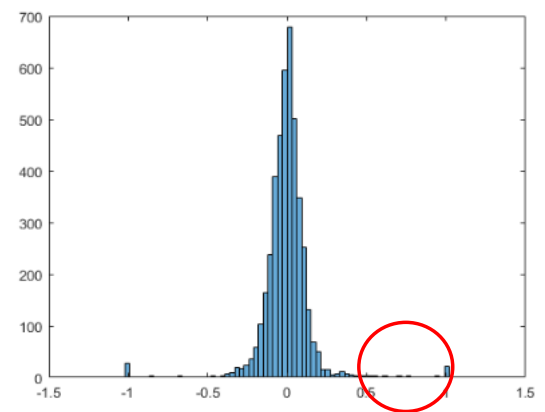

(b)

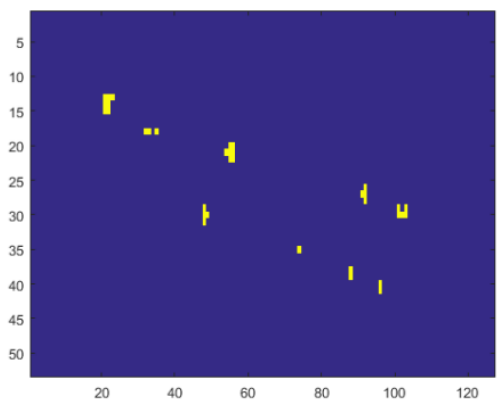

(c)

Figure 3. The error map: (a) Visualization of the error map matrix; Red circle: pixels surrounding the detectable roof detail; Blue circle: missing details; (b) Histogram of error map values; Red circle: missing roof detail; (c) Mask of missing roof details



Figure 4. Types of missing roof details; Red circle: roof detail having considerable altitude; Blue circle: roof detail having negligible altitude; detail altitude can be noted from the shadow length

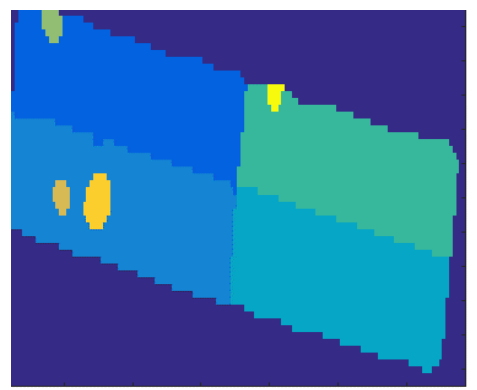

(a)
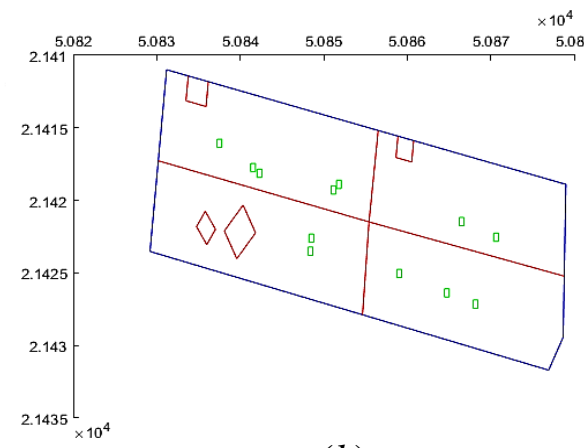

(b)

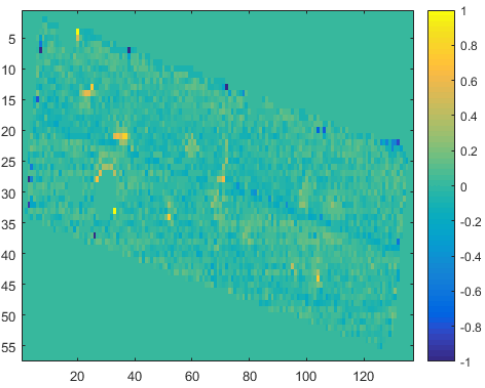

(c)

Figure 5. (a) Building label image of improved roof segmentation (the colours present the plane numbers); (b) 2D building model (outer building boundary is in blue, inner roof plane boundaries are in red and missing roof details are in green); $(c)$ Error map after re-segmentation 


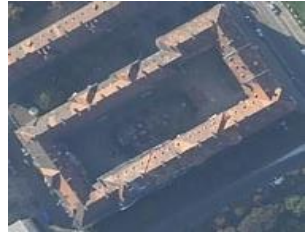

(a)

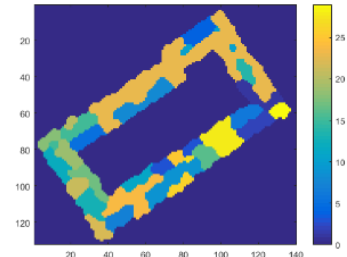

(b)

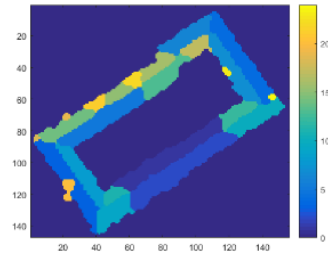

$(c)$

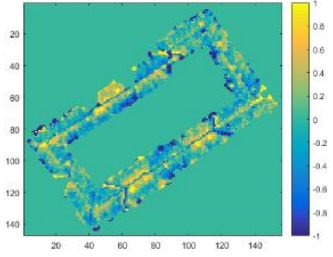

$(d)$

Figure 6. (a) Building aerial image; (b) Building label image; case of mis-segmented building; $(c)$ Building label image after improvement; $(d)$ Visualization of error map matrix after improvement (the building is in Strasbourg city in France)

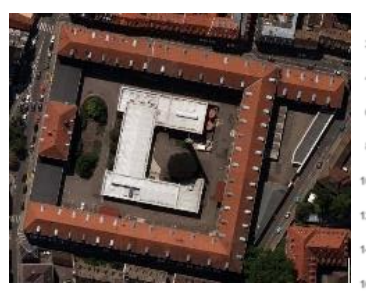

(a)

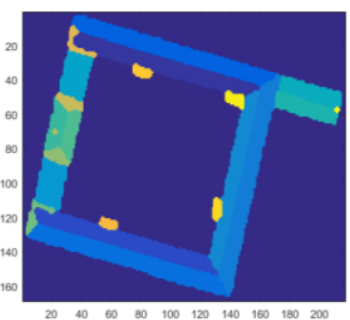

(b)

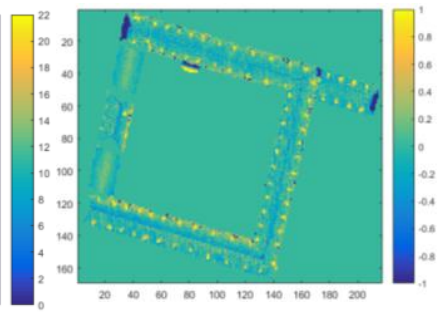

(c)

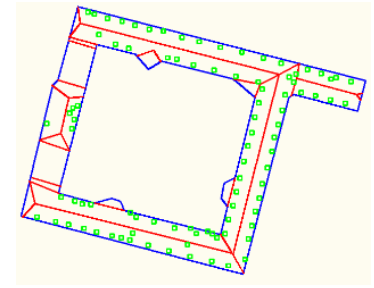

$(d)$

Figure 7. Successful segmented building; (a) Aerial image; $(b)$ Building label image; $(c)$ Error map; (d) 2D building model (the building is in Strasbourg city in France)

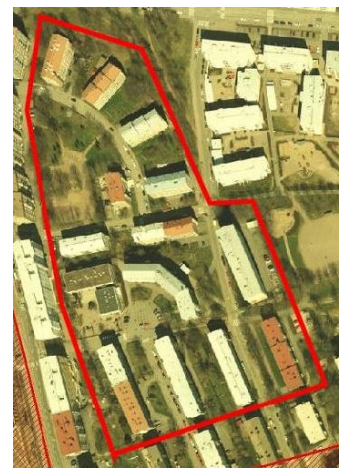

(a)

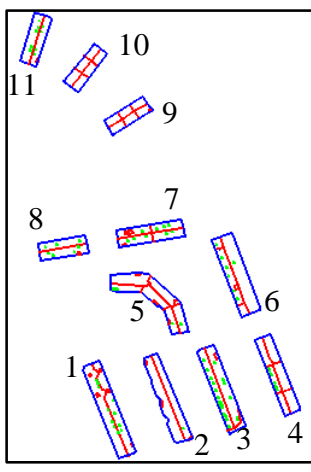

(b)

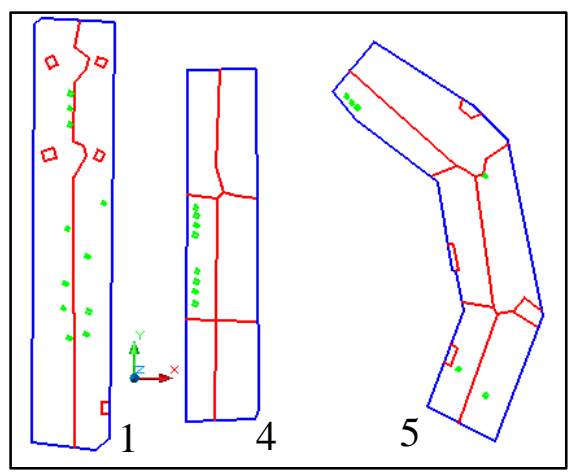

(c)

Figure 8. Final result of automatic 2D building modelling of Hermanni site; $(a)$ Aerial image; $(b)$ 2D building models; $(c)$ Zooming of 3 building models (blue: the building outer boundaries; red: the inner roof boundaries; green: missing details) 


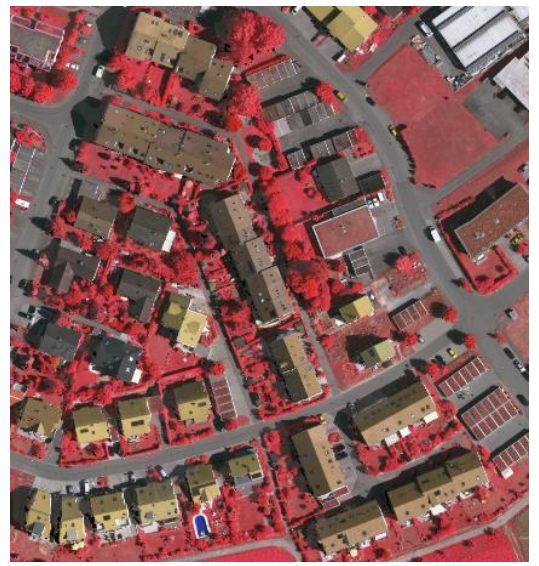

(a)

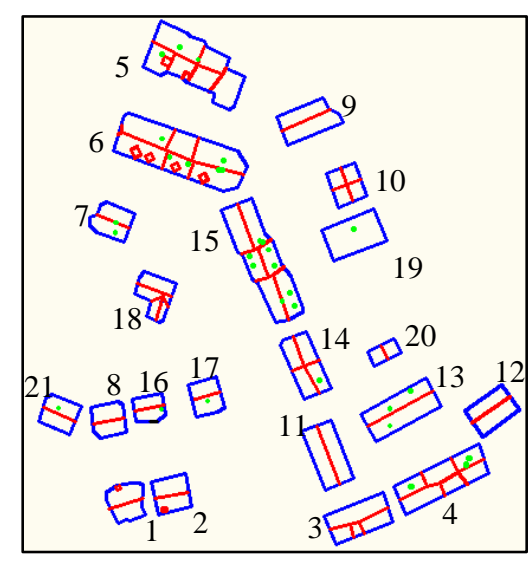

(b)

Figure 9. Final result of automatic 2D building modelling of Vaihingen site number 1; (a) Aerial image; (b) 2D building models (blue: the building outer boundaries; red: the inner roof boundaries; green: missing details)



(a)

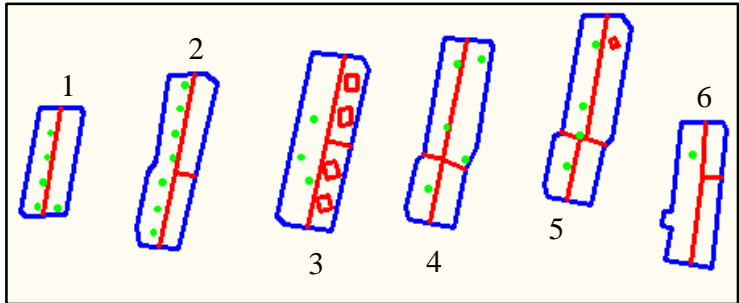

(b)

Figure 10. Final result of automatic 2D building modelling of Vaihingen site number 2; $(a)$ Aerial image; $(b)$ 2D building models (in blue: the building outer boundaries; in red: the inner roof boundaries; in green: missing details)

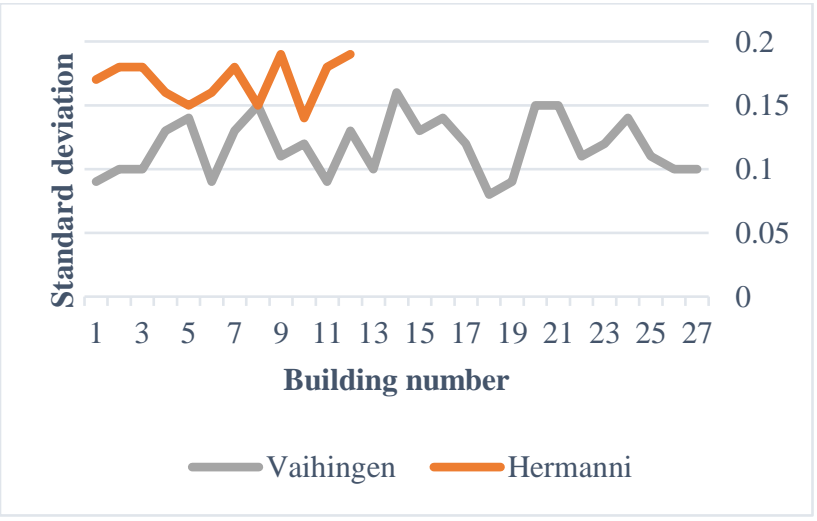

Figure 11. Standard deviations of 3D building models 